\title{
Computer-assisted Development of ad hoc Methodology for Monitoring of Inorganic Cations in Surface Water Using Gradient Elution Ion Chromatography
}

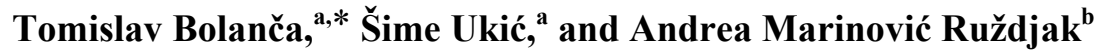 \\ ${ }^{a}$ Faculty of Chemical Engineering and Technology, University of Zagreb, Marulićev trg 20, 10000 Zagreb, Croatia \\ ${ }^{\mathrm{b}}$ Central Water Management Laboratory, Croatia Waters, Ulica grada Vukovara 220, 10000 Zagreb, Croatia
}

RECEIVED JULY 21, 2010; REVISED MAY 5, 2011; ACCEPTED MAY 9, 2011

\begin{abstract}
This work focuses on development of ad hoc methodology for monitoring of sodium, ammonium, potassium, magnesium and calcium ions in rivers Ilova and Kutinica (southeastern part of Croatia) since often their composition significantly differs from the average (concentration of components and/or matrix). Therefore fast and reliable method development has to be performed in order to meet criteria of the monitoring methodology performance characteristic. Computer assisted routine for prediction of chromatographic signal, based on a transfer of isocratic elution information into a gradient elution environment, was used in combination with several in-house developed optimization criteria. It is shown that algorithm used for prediction of ion-chromatographic signal offers sufficient information (in comparison to actual measured chromatographic signal) to be applied in global optimization process. Calculated optimal gradient incorporates linearly decreasing, increasing and isocratic part of the eluent concentration time-profile, utilizing full potential of the gradient separation. Developed method was validated proving that it meets criteria for river Ilova and Kutinica monitoring purposes. Moreover, this results show that used computer assisted optimization routine can be successfully applied for fast and reliable ad hoc method development in ion chromatography. (doi: 10.5562/cca1736)
\end{abstract}

Keywords: ion chromatography, inorganic cation, surface water, optimization

\section{INTRODUCTION}

The analysis of inorganic ions in drinking, ground and surface waters still remain the single most widely used application of ion chromatography (IC).$^{1-3}$ IC provides a straightforward method for the simultaneous analysis of alkali and alkaline earth cations (plus ammonia) in water, as described in ISO Method 14911-1. ${ }^{4}$ A key benefit of this approach is the ability to determine ammonia in complex samples that contain both: inorganic cations and organic amines, as the latter compounds can interfere with the conventional colorimetric or ion selective electrode methods used for ammonia analysis. The problem arises if samples with significantly different composition (matrix and/or analyte components concentration) have to be analyzed. These deviations can be result of natural phenomenon (rainfall, snowing, etc.) or environmental incident because of human activities. In either case, the standard or accredited methodology for analysis of river water has to be modified and later on validated to prove that can fit the purpose. The modified method, referred as "ad hoc" method, should be able to carry out exploratory studies in the short term, or for a short run of test materials. Since, minimal time is usually available to modify and validate performance characteristics, it is necessary to rely largely on the computer assisted method development and, due to the most probably tight schedule, gradient elution ion chromatography.

The computer assisted method development in general relies on retention modeling. A large number of retention models were explained for isocratic IC elution. $^{5-13}$ Gradient elution retention model is harder to develop due to a fact that far more variables need to be modeled in order to present sufficient gradient combinations. Artificial neural networks (ANN) have modeled the retention behavior of inorganic anions, in relation with slope of linear gradient elution curve and starting time of gradient program. ${ }^{14,15}$ The ANN gradient elution retention models have excellent accuracy and precision but gradient elution experimental data, which has been used for modeling procedure, cannot be used for modeling of various gradient programs. The one possibility to overcome that shortcoming is to use isocratic experimental data for gradient elution retention modeling. ${ }^{16,17}$

\footnotetext{
* Author to whom correspondence should be addressed. (E-mail: tomislav.bolanca@fkit.hr)
} 


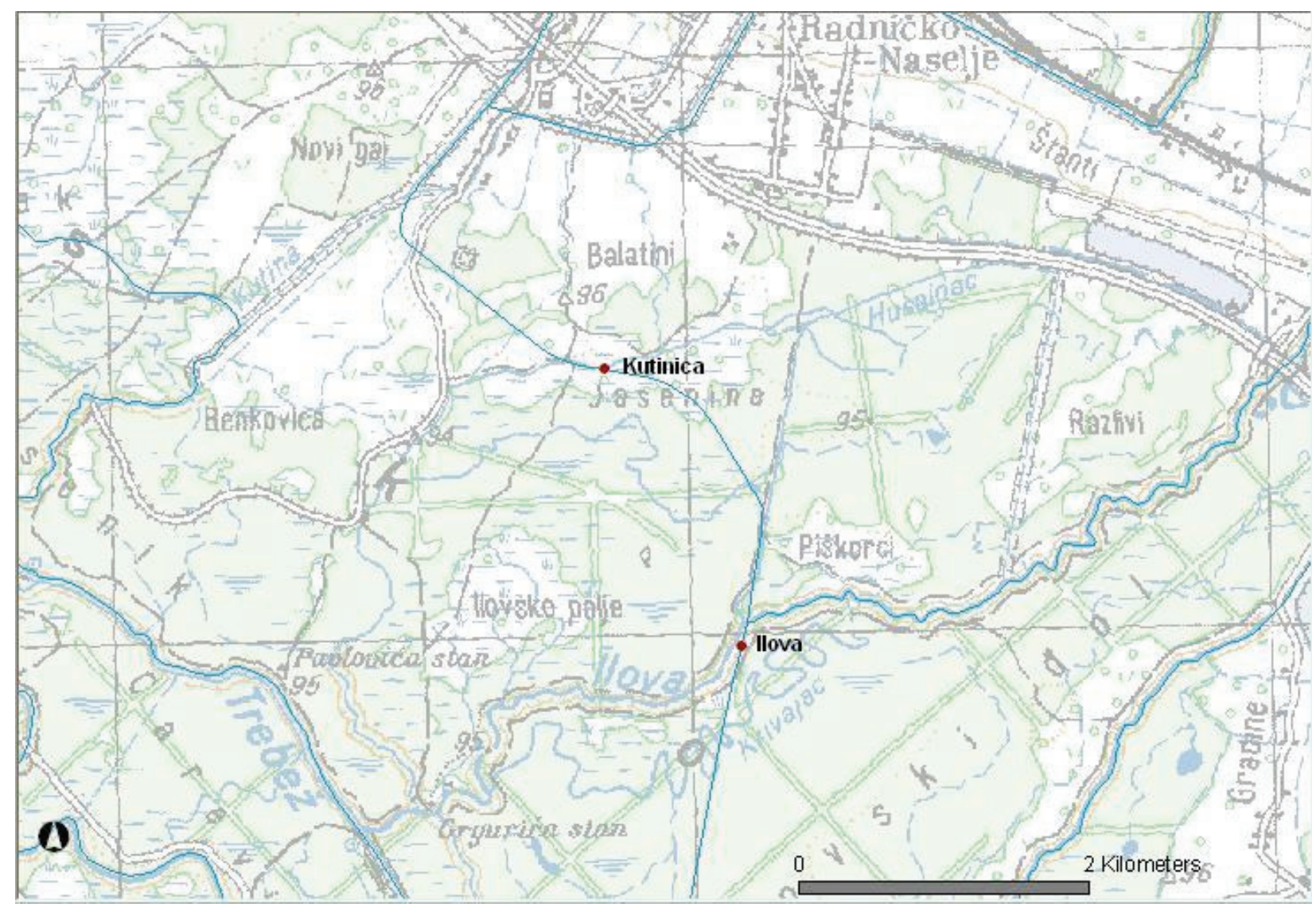

Figure 1. Monitoring locations of river Kutinica and river Ilova.

Finally, developed retention models than can be used for resolution and peak asymmetry predication even the peak shape modeling if necessary. ${ }^{18,19}$

The aim of this work is application of computerassisted methodology in rapid method development process for IC determination of inorganic cations in surface water. Gradient elution retention model was used to predict resolution and peak asymmetry. These parameters indicate the ability of method for quantitative determination of analyte, and therefore were needed for determination of optimal gradient elution profile. Final stage includes application of peak shape model to predict the chromatographic signal and its comparison with actual experiment. Performance characteristics of developed method were extensively tested by applying validation procedure.

\section{EXPERIMENTAL}

\section{Monitoring Locations}

$97 \mathrm{~km}$ long river Ilova rises in the southeastern part of Croatia at $200 \mathrm{~m}$ above sea level. Throughout its course, river Ilova is a lowland medium-sized watercourse in the silicate surface. Channel Kutinica-Ilova connects river Ilova with the small river Kutinica. Watercourse of river Kutinica is exposed to the wastewater inflow of Petrokemija d.o.o, Kutina, fertilizer factory. It is obvious that water quality of both rivers: Kutinica (after the fertilizer factory inflow) and Ilova (after river Kutinica inflow), are included in water monitoring process. The previous monitoring results showed significant and variable presence of monitoring components, which occurs in random manner, depending mostly on different production conditions of fertilizer factory. These circumstances make ad hoc method development process of high significance for the region. In this work, two different locations were tested: one after the wastewater inflow in river Kutinica and the other after the Kutinica inflow in river Ilova. The exact locations are shown in Figure 1.

\section{Optimization Methodology}

For optimization of IC method, an approach with gradient model based on isocratic data (iso-to-grad model) was used. This approach, taken from literature, ${ }^{16,19}$ is based on summation of contributions of small timeintervals inside which the retentions were calculated by use of experimentally obtained isocratic models. The isocratic models were $2^{\text {nd }}$ order polynomial correlations of analyte retention coefficient logarithm, $\log k$, and 
Table 1. Description of sodium, ammonium, potassium, magnesium and calcium retention models for isocratic elution

\begin{tabular}{|c|c|c|c|c|c|}
\hline \multirow{2}{*}{ Isocratic modelling } & \multicolumn{5}{|c|}{$\log k=a_{0}+a_{1} \log c_{\mathrm{MSA}}+a_{2} \log ^{2} c_{\mathrm{MSA}}$} \\
\hline & Sodium & Ammonium & Potassium & Magnesium & Calcium \\
\hline & \multicolumn{5}{|c|}{ Model parameters for retentions of $50 \%$ height at peak fronting side } \\
\hline$a_{0}$ & 2.1608 & 2.3865 & 2.4289 & 4.3000 & 4.6233 \\
\hline$a_{1}$ & -1.5278 & -1.6634 & -1.4243 & -2.7071 & -2.9418 \\
\hline$a_{2}$ & 0.1117 & 0.1708 & 0.0977 & 0.1670 & 0.2482 \\
\hline \multirow[t]{2}{*}{$R^{2}$} & 0.9999 & 0.9999 & 0.9999 & 0.9999 & 0.9999 \\
\hline & \multicolumn{5}{|c|}{ Model parameters for retentions of peak maximum } \\
\hline$a_{0}$ & 2.1795 & 2.3857 & 2.4386 & 4.3582 & 4.6607 \\
\hline$a_{1}$ & -1.5535 & -1.6614 & -1.4366 & -2.7936 & -2.9952 \\
\hline$a_{2}$ & 0.1241 & 0.1730 & 0.1036 & 0.2015 & 0.2693 \\
\hline \multirow[t]{2}{*}{$R^{2}$} & 0.9999 & 0.9999 & 0.9999 & 0.9999 & 0.9999 \\
\hline & \multicolumn{5}{|c|}{ Model parameters for retentions of $50 \%$ height at peak tailing side } \\
\hline$a_{0}$ & 2.1901 & 2.3820 & 2.4512 & 4.4210 & 4.6945 \\
\hline$a_{1}$ & -1.5698 & -1.6566 & -1.4480 & -2.8648 & -3.0082 \\
\hline$a_{2}$ & 0.1339 & 0.1753 & 0.1089 & 0.2266 & 0.2717 \\
\hline$R^{2}$ & 0.9999 & 0.9999 & 0.9999 & 0.9999 & 0.9999 \\
\hline
\end{tabular}

eluent concentration $\operatorname{logarithm}, \log c$. Experimental design used in obtaining the modeling data was based on full factorial methodology and is shown in Figure 2. Inside each time interval the retention coefficient can be assumed constant, and is calculated as the average of $k$ values at the integral boundaries; the latter are calculated by use of the isocratic model. The coefficients of polynomial isocratic models are given in Table 1 along with related $R^{2}$ values, showing models' good predictive abilities.

For selection of optimal gradient elution profile, a large but finite number of gradient eluent-profiles was generated (so called profile domain) and tested in the following manner. ${ }^{20}$ Four criteria were used for quicker scanning of profile domain. The first criterion was comparing resolution values of the neighboring isocratic eluent-profiles from profile domain. It selects two most distant isocratic profiles with unacceptable $R_{\mathrm{S}}$ value $\left(R_{\mathrm{S}}<2\right)$ for the same analytes and eliminates all other domain profiles: gradient or isocratic, which are lying between them. The second criterion was searching the domain for the isocratic profiles with all acceptable $R_{\mathrm{S}}$ values. If one or more existed, it selects the one with highest eluent concentration, and all domain profiles bellowed it were expelled from the domain. The third criterion was based on the nature of iso-to-grad model. This criterion monitored the contributions of timeintervals inside every domain profile that had an eluent concentration change. If a sequence of time-intervals that provides unsatisfactory separation was detected, all domain profiles that had included the same sequence were, therefore, excluded from the domain. Fourth cri- terion select the shortest chromatography run if more than one optimal profile remained after the treatment of first three criteria.

\section{Instrumentation}

Experiments were performed on Dionex ICS-3000 chromatography system (Sunnyvale, CA, USA). The system consisted of a dual pump module with an isocratic pump with vacuum degas option and gradient mixer (GM-3, $4 \mathrm{~mm}$ ), eluent generator module with degas unit and trap column (CR-TC II) and detector/ chromatography module with a conductivity detector and dual temperature zone configuration. Carboxylatebased CS16 $(5 \times 250 \mathrm{~mm})$ cation separation column and

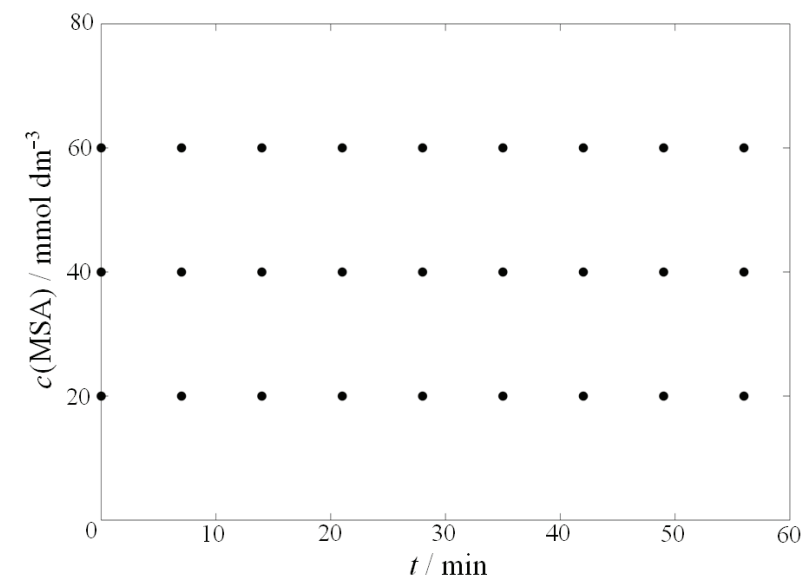

Figure 2. Isocratic elution experimental design used in optimization process. 

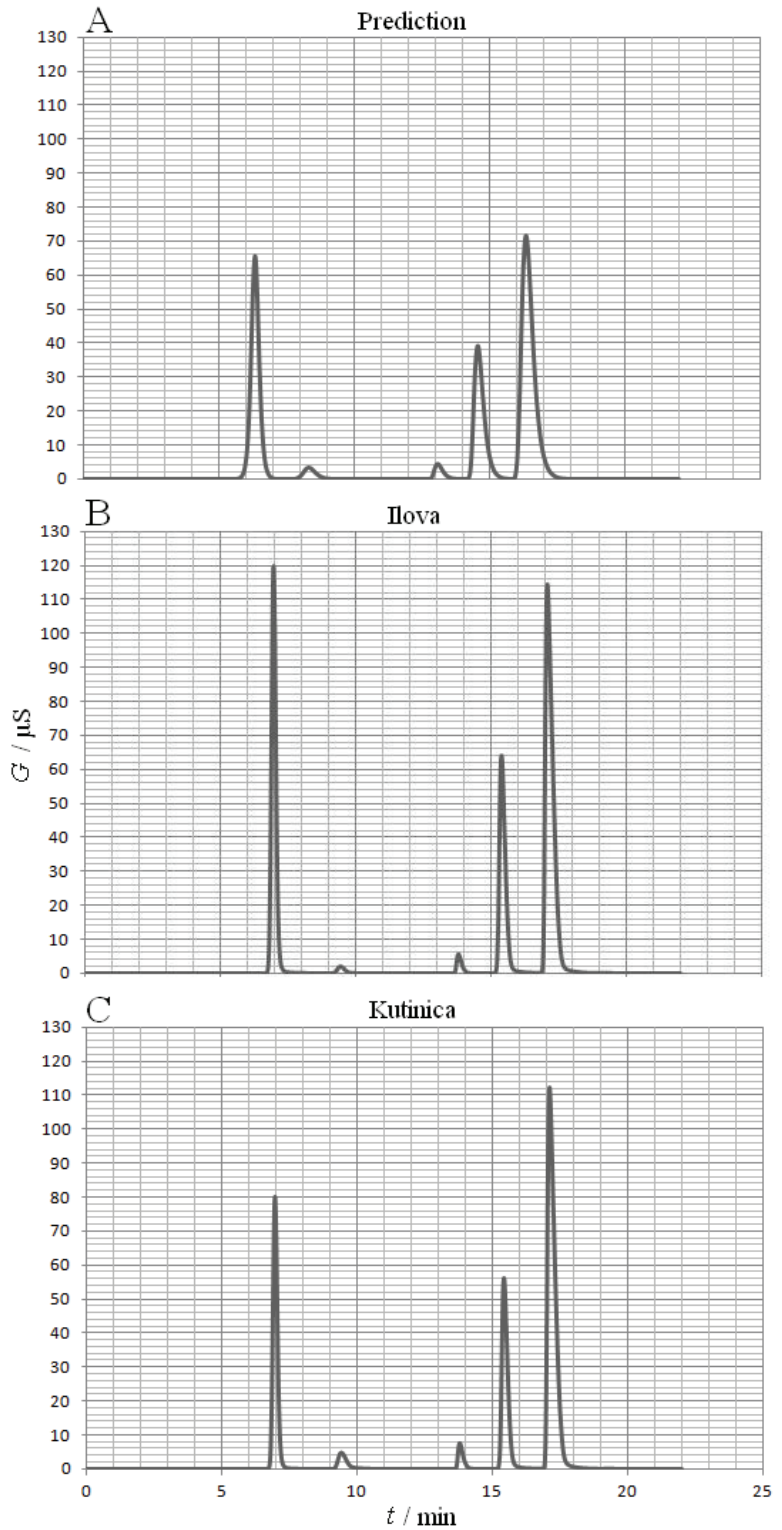

Figure 3. Simulated (A) and measured (B, C) ion chromatographic separation obtained by using optimal gradient profile.

CG16 $(5 \times 50 \mathrm{~mm})$ cation guard column were used for separation of common inorganic cations. The eluent was methanesulfonic acid solution. For decreasing of background noise and increasing of outgoing analytical signal, CSRS Ultra II 4-mm electrolytic suppressor (working in recycle mode) was used. All experimental measurements were performed using $50 \mu \mathrm{L}$ sample loop volume, eluent flow rate of $1.0 \mathrm{~mL} / \mathrm{min}$, and temperature of $40{ }^{\circ} \mathrm{C}$. The whole system was computercontrolled by Chromeleon 6.80 SP4 Build 2361 (130805) software.

\section{Reagents and Solutions}

Water samples were collected in $2 \mathrm{~L}$ polyethylene containers and filtered through Whatman $0.45 \mu \mathrm{m}$ cellulose

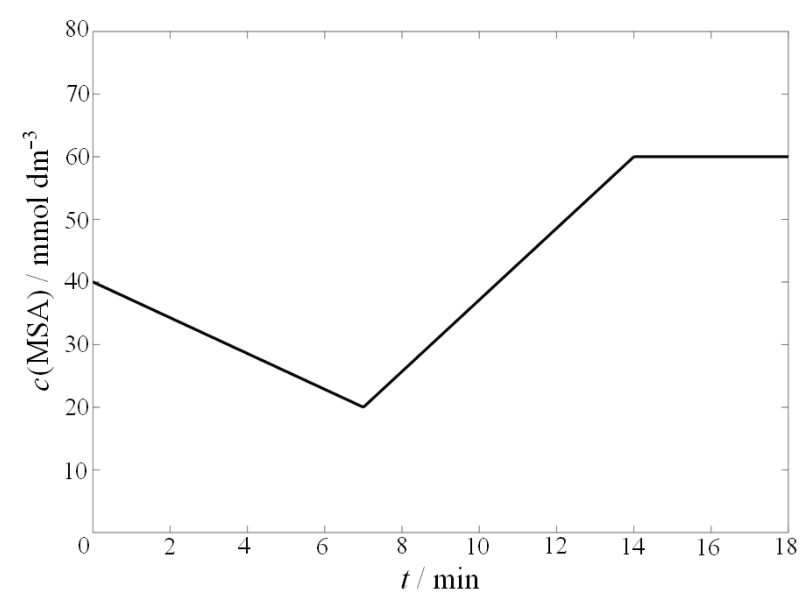

Figure 4. Optimal gradient profile obtained by using computer-assisted optimization methodology.

nitrate membrane filters (Whatman, Springfield Mill, England) prior to injection. The first $100 \mathrm{~mL}$ of filtered sample was discarded.

Concentration values of cations found in surface water samples were used as guidelines in selecting calibration ranges. The used calibration ranges were: sodium $20-60 \mathrm{mg} / \mathrm{L}$, ammonium $1-3 \mathrm{mgN} / \mathrm{L}$, potassium 2-6 mg/L, magnesium 7-21 mg/L, and calcium 25-75 $\mathrm{mg} / \mathrm{L}$. Standard solutions of sodium, ammonium, potassium, magnesium (all Kemika, Zagreb, Croatia), and calcium (Fluka, Taufkirchen, Germany) were prepared by dissolving appropriate amounts of analytical reagent grade salts in Milli-Q water (Millipore, Bedford, MA, USA; $0.055 \mu \mathrm{Scm}^{-1}$ ). These solutions were diluted to give the multi-cation solutions required. Working eluent solutions were prepared on-line by appropriate dilution of methanesulfonic acid (MSA) cartridge solution (Dionex) with Milli-Q water.

\section{RESULTS AND DISCUSSION}

Figure 3 presents the simulated and measured chromatograms for analysis of inorganic cations in surface waters. One can observe that simulated peaks are slightly shifted towards the lower retention region. The reason for this is most probably definition of void peak retention, which is in this work assumed constant and is curtail for gradient elution signal prediction. Modeling void peak retention under gradient elution would significantly increase experimental effort, putting in question justification of the applied procedure. Analogue conclusion can be made if the peak shape prediction capabilities are discussed. However, from the method development point of view, the predicted chromatograms bring more than enough useful information that can be later on successfully used in global optimization process. 
Table 2. Calibration curve parameters calculated for IC determination of sodium, ammonium, potassium, magnesium and calcium in rivers Ilova and Kutinica

\begin{tabular}{|c|c|c|c|c|c|c|c|c|}
\hline \multirow{2}{*}{ Linearity } & \multicolumn{2}{|l|}{ Value } & \multicolumn{3}{|c|}{ Slope $(\mu \mathrm{S} \min \mathrm{L} / \mathrm{mg})$} & \multicolumn{3}{|c|}{ Intercept $(\mu \mathrm{S} \min )$} \\
\hline & Range (mg/L) & $R^{2}$ & Value & Lower $95 \%$ & Upper $95 \%$ & Value & Lower $95 \%$ & Upper $95 \%$ \\
\hline Sodium & $20.00-60.00$ & 0.9998 & 0.6106 & 0.6082 & 0.6130 & 0.0195 & -0.0824 & 0.1215 \\
\hline Ammonium & $1.50-4.50$ & 0.9997 & 0.4942 & 0.4916 & 0.4969 & 0.3837 & 0.3754 & 0.3920 \\
\hline Potassium & $2.00-6.00$ & 0.9997 & 0.4059 & 0.4037 & 0.4081 & -0.0227 & -0.0319 & -0.0134 \\
\hline Magnesium & $7.00-21.00$ & 0.9997 & 1.1003 & 1.0948 & 1.1057 & 0.2946 & 0.2131 & 0.3762 \\
\hline Calcium & $25.00-75.00$ & 0.9999 & 0.7233 & 0.7209 & 0.7257 & 0.2724 & 0.1429 & 0.4020 \\
\hline
\end{tabular}

Figure 4 shows optimal gradient profile obtained by computer-assisted development of ad hoc methodology. Although optimal gradient profile might seem simple, deeper insight could reveal its capabilities. The first part of the separation process was performed using negative linear gradient profile. This particular profile apparently increased analysis time, but it provided satisfactory resolution of two first-eluted components, without sacrificing resolution of the remaining components, and actually, it led to the shorter analysis time. Next part of the gradient elution profile could be considered as typical one - a classical linear increase of the competing ion concentration over the certain time period. This part decreased the analysis time by lowering time between elution of last three components and the earlier eluted ones. The last part of the gradient profile presents classical isocratic elution. The reason for this is that positive gradient would decrease required resolution and negative gradient would increase the analysis time. In gener-

Table 3. Lack-of-fit calculations for calibration curves related to IC determination of sodium, ammonium, potassium, magnesium, and calcium in rivers Ilova and Kutinica

\begin{tabular}{lllcc}
\hline \multirow{2}{*}{ Linearity } & \multicolumn{4}{c}{ Lack-of-fit test } \\
\cline { 2 - 5 } & $M S_{\mathrm{PE}}$ & $M S_{\mathrm{LOF}}$ & $F_{\text {crit }}$ & $F$ \\
\hline Sodium & 0.0114 & 0.0307 & 2.8387 & 2.6905 \\
Ammonium & $8.47506 \cdot 10^{-5}$ & $7.7684 \cdot 10^{-5}$ & 2.8387 & 0.9166 \\
Potassium & 0.0001 & 0.0002 & 2.8387 & 1.5077 \\
Magnesium & 0.0077 & 0.0145 & 2.8387 & 1.8836 \\
Calcium & 0.0185 & 0.0493 & 2.8387 & 2.6651 \\
\hline
\end{tabular}

al, gradient profiles obtained by using computer optimization methodology typically consist of positive linear parts or positive steps. This means that gradient elution benefits might be not utilized entirely if using these profiles. However, the good separation is only one factor (might be the curtail one) that has to be considered in method development process. Therefore, further evaluation of performance characteristic has to follow.

Tables 2 and 3 present the results of linearity tests. It can be seen that squares of correlation coefficient $\left(R^{2}\right)$ are ranged between 0.9997 and 0.9999 proving that high correlation between concentration and peak area for all investigated cations exists. The good sensitivity of the method is expressed by slope of the calibration curve and the limits of lower and upper $95 \%$ confidence intervals are presented (Table 2). The lack-of-fit test, based on analysis of the variance (ANOVA) of the residuals from the regression line, was applied to test the validity of selected calibration models (Table 3 ). Testing for lack of fit of the experimental points to the regression line is an important step in linear regression. When lack of fit exists, standard deviations for both regression line coefficients are overestimated, and this gives rise, for instance, to confidence intervals that are too large. If these confidence intervals are then used in hypothesis tests, systematic error may not be detected so there is a greater probability of committing a $\beta$ error. The $F$ values obtained for lack-of-fit test prove that lack-of-fit term is not significant within $95 \%$ confidence limit for pure experimental error and consequently the straight line model is adequate to describe the relationship between concentration and peak area for all

Table 4. Systematic error estimation for IC determination of sodium, ammonium, potassium, magnesium, and calcium in rivers Ilova and Kutinica

\begin{tabular}{|c|c|c|c|c|c|c|c|}
\hline \multirow{2}{*}{ Linearity } & \multirow{2}{*}{$R^{2}$} & \multicolumn{3}{|c|}{ Slope } & \multicolumn{3}{|c|}{ Intercept $(\mathrm{mg} / \mathrm{L})$} \\
\hline & & Value & Lower $95 \%$ & Upper $95 \%$ & Value & Lower $95 \%$ & Upper $95 \%$ \\
\hline Sodium & 0.9998 & 1.0000 & 0.9961 & 1.0039 & $6.39488 \cdot 10^{-14}$ & -0.16692 & 0.16692 \\
\hline Ammonium & 0.9997 & 1.0000 & 0.9947 & 1.0053 & $8.88178 \cdot 10^{-16}$ & -0.01675 & 0.01675 \\
\hline Potassium & 0.9997 & 1.0000 & 0.9946 & 1.0054 & 0.00000 & -0.02273 & 0.02273 \\
\hline Magnesium & 0.9997 & 1.0000 & 0.9950 & 1.0050 & $-1.77636 \cdot 10^{-15}$ & -0.07413 & 0.07413 \\
\hline Calcium & 0.9999 & 1.0000 & 0.9966 & 1.0034 & $5.68434 \cdot 10^{-14}$ & -0.17909 & 0.17909 \\
\hline
\end{tabular}


Table 5. Recovery of IC determination of sodium, ammonium, potassium, magnesium and calcium in rivers Ilova and Kutinica

\begin{tabular}{lcc}
\hline \multirow{2}{*}{ Accuracy } & \multicolumn{2}{c}{ Recovery (\%) } \\
\cline { 2 - 3 } & Value & RSD \\
\hline Sodium & 100.00 & 0.20 \\
Ammonium & 100.00 & 0.17 \\
Potassium & 100.02 & 0.26 \\
Magnesium & 100.03 & 0.29 \\
Calcium & 100.00 & 0.16 \\
\hline
\end{tabular}

investigated cations (Table 3). Furthermore, presence of the systematic error was tested by performing replicate measurements at five different concentrations levels and relationship between amount found (y) against amount added $(x)$ were investigated (Table 4 ). It can be seen that intercepts and slopes are not statistically (confidence $95 \%$ ) different form the theoretical ones ( 0 and 1 respectively) and that squares of correlation coefficients have values not lower that 0.9997 for all investigated cases. This proves that there is no systematic error (proportional and absolute) in developed method. Values for recovery fall into interval 100.00-100.03, with maximal relative standard deviation (RSD) of $0.29 \%$, what proves particularly good recovery of the method (Table 5). The precision related calculations are presented in Table 6. It can be seen that RSD is less than $0.33 \%$ for all investigated cases showing good precision of the method developed for determination of sodium, ammonium, potassium, magnesium and calcium in rivers Ilova and Kutinica.

\section{CONCLUSIONS}

This work describes computer-assisted development of ad hoc methodology for monitoring of inorganic cations in rivers Ilova and Kutinica using gradient elution IC. It is shown that IC signal prediction slightly deviate from the actual measured signal in both retention time and peak shape parameter due to application of constant void time in the calculation routines. Still, virtual chromatography brings enough information to be successfully used in optimization procedure. Applied optimization algorithms resulted in gradient profile that incorporates linear decreasing and increasing of eluent concentration followed by an isocratic part of the separation, offering complete unitization of gradient elution benefits. Performance characteristics of developed method included linearity test (with lack-of-fit), systematic error test, method recovery and repeatability estimation, proving that developed method can meet criteria for routine application in monitoring rivers' Ilova and Kutinica
Table 6. Repeatability of IC determination of sodium, ammonium, potassium, magnesium and calcium in rivers Ilova and Kutinica $(n=6)$

\begin{tabular}{lc}
\hline Accuracy & RSD $(\%)$ \\
\hline Sodium & 0.20 \\
Ammonium & 0.33 \\
Potassium & 0.29 \\
Magnesium & 0.28 \\
Calcium & 0.19 \\
\hline
\end{tabular}

quality. Moreover, this proves that computer assisted methodology offers fast and reliable tool for ad hoc method development in ion chromatography.

\section{REFERENCES}

1. P. R. Haddad and P. E. Jackson, Ion Chromatography: Principles and Applications, J. Chromatogr. Library, Vol. 46, Elsevier, Amsterdam, 1990.

2. J. Weiss, Handbook of Ion Chromatography, Wiley VCH, Verlag, 2004.

3. J. S. Fritz and D. T. Gjerde, Ion Chromatography, $3^{\text {rd }}$ ed., WileyVCH, New York, 2000.

4. ISO 14911, Water quality - Determination of dissolved $\mathrm{Li}^{+}, \mathrm{Na}^{+}$ $\mathrm{NH}_{4}^{+}, \mathrm{K}^{+}, \mathrm{Mn}^{+}, \mathrm{Ca}^{2+}, \mathrm{Mg}^{2+}, \mathrm{Sr}^{2+}$, and $\mathrm{Ba}^{2+}$ using ion chromatography -Method for water and wastewater, ISO, Geneva, 1998.

5. P. Hajos, O. Horvath, and V. Denke, Anal. Chem. 67 (1995) 434-441.

6. L. R. Snyder and J. W. Dolan, in: P. R. Brown and E. Grushka (Eds.), Advances in Chromatography, Vol. 38, Marcel Dekker Inc., New York, 1998, pp.115-185.

7. P. Hajos, O. Horvath, and G. Revesz, in: P. R. Brown and E. Grushka (Eds.), Advances in Chromatography, Vol. 39, Marcel Dekker Inc., New York, 1998, pp.313-350.

8. J. Stahlberg, J. Chromatogr. A 855 (1999) 3-55.

9. J. E. Madden and P. R. Haddad, J. Chromatogr. A 829 (1998) 65-80.

10. J. E. Madden and P. R. Haddad, J. Chromatogr. A 850 (1999) 29-41.

11. J. E. Madden, N. Avdalović, P.E. Jackson, and P. R. Haddad, J Chromatogr A 837 (1999) 65-74.

12. G. Sacchero, M. C. Bruzzoniti, C. Sarzanini, E. Menstati, and H. J. Melting, J. Chromatogr. A 799 (1999) 35-45.

13. T. Bolanča, Š. Cerjan-Stefanović, M. Regelja, H. Regelja, and S. Lončarić, J. Chromatogr. A 1085 (2005) 74-85.

14. J. E. Madden, N. Avdalovic, P. R. Haddad, and J. Havel, J. Chromatogr. A 910 (2001) 173-179.

15. T. Bolanča, Š. Cerjan-Stefanović, M. Luša, H. Regelja, and S. Lončarić, Chemom. Intell. Lab. Syst. 86 (2007) 95-101.

16. G. Vivó-Truyols, J. R. Torres-Lapasió, M. C. Garcia-AlvarezCoque, J Chromatogr A 1018 (2003) 169-181.

17. T. Bolanča, Š. Cerjan-Stefanović, M. Luša, M. Rogošić, and Š. Ukić, J Chromatogr A 1121 (2006) 228-235.

18. T. Bolanča, Š. Cerjan-Stefanović, Š. Ukić, M. Rogošić, and M. Luša, J. Liq. Chrom. Rel. Technol. 32 (2009) 1373-1391.

19. T. Bolanča, Š. Cerjan-Stefanović, Š. Ukić, M. Rogošić, and M. Luša, J. Sep. Sci. 32 (2009) 2877-2884.

20. Š. Ukić, T. Bolanča, and M. Rogošić, J. Sep. Sci. 34 (2011) 780-788. 